\title{
Adubação nitrogenada na miniestaquia de erva-mate
}

\author{
Manoela Mendes Duarte $\circledast^{1^{*}}$ Mônica Moreno Gabira $\odot^{1}$ Jéssica de Cássia Tomasi ${ }^{1}$ Leandro \\ Marcolino Vieira $\oplus^{1}$ Natália Saudade de Aguiar $\oplus^{1}$ Renata de Almeida Maggioni $\oplus^{1}$ Erika Amano $\oplus^{1}$ \\ Marcelo Lazzarotto $\circledast^{2}$ Antonio Carlos Nogueira $\circledast^{1}$ Ivar Wendling $\circledast^{2}$
}

${ }^{1}$ Universidade Federal do Paraná, Av. Pref. Lothário Meissner, 632, Jardim Botânico, Campus III, CEP 80210-170, Curitiba, PR, Brasil. ${ }^{2}$ Empresa Brasileira de Pesquisa Agropecuária - Embrapa Florestas, Estrada da Ribeira, Km 111, CEP 83411-000, Colombo - PR, Brasil.

\section{Original Article \\ *Corresponding author: \\ manu- \\ florestal@hotmail.com}

Palavras-chave:

Ilex paraguariensis

Minijardim clonal

Rizogênese

Propagação vegetativa

Keywords:

Ilex paraguariensis

Clonal mini-garden

Rhizogenesis

Vegetative propagation

Received in

2020/01/22

Accepted on

2020/04/04

Published in

2020/06/30

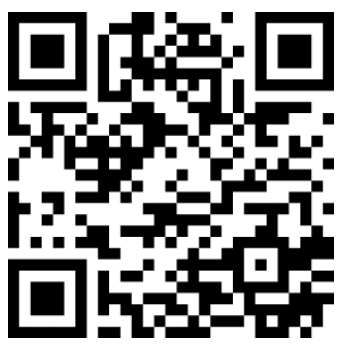

DOI:

https://doi.org/10.34062/afs. v7i2.9716

\section{(cc) BY}

RESUMO: Objetivou-se avaliar a influência de doses de nitrogênio em solução nutritiva na rizogênese de miniestacas de dois clones (EC22 e EC40) de Ilex paraguariensis coletadas em diferentes épocas do ano. As minicepas foram conduzidas em sistema semi-hidropônico, recebendo via gotejamento solução com macro e micronutrientes com cinco doses de nitrogênio: 104, 206, 380, 760 e 1142 $\mathrm{mg} \mathrm{L}^{-1}$. As brotações foram coletadas em quatro épocas do ano, sendo preparadas miniestacas com $8 \pm 1 \mathrm{~cm}$, mantendo de duas a três folhas inteiras na porção superior. Após tratamento com AIB (3000 mg L-1), as miniestacas foram plantadas em tubetes contendo substrato comercial e permaneceram em casa de vegetação com temperatura e umidade controladas. Após 120 dias do plantio foram avaliados enraizamento, número médio de raízes/miniestaca, comprimento médio de raízes, miniestacas vivas e mortas, presença de calos, miniestacas com raiz e calo, emissão de novas brotações e manutenção de folhas. $\mathrm{O}$ experimento foi conduzido em delineamento inteiramente casualizado, em esquema fatorial ( 2 clones x 5 doses de $\mathrm{N}$ ), em parcelas subdivididas no tempo (épocas de coleta), sendo as variáveis submetidas à ANOVA e Tukey. Houve influência dos fatores para todas as variáveis analisadas, sendo que os clones responderam de maneiras distintas às doses de nitrogênio em cada coleta, indicando a importância das condições ambientais e nutricionais nas quais as minicepas são mantidas. Recomenda-se a aplicação de 104 a $380 \mathrm{mg} \mathrm{L}^{-1}$ de nitrogênio na solução nutritiva.

\section{Nitrogen fertilization in yerba mate mini-cutting}

\begin{abstract}
This study aimed to evaluate the influence of nitrogen doses used in nutrient solution on the rhizogenesis of mini-cuttings of two Ilex paraguariensis clones (EC22 and EC40 clones), collected at different harvesting seasons. The ministumps were conducted in a semi-hydroponics system with drip irrigation, receiving five nitrogen levels: 104, 206, 380, 760 and $1142 \mathrm{mg} \mathrm{L}^{-1}$. The shoots with $8 \pm 1 \mathrm{~cm}$ were collected at four harvesting seasons during the year, keeping two to three whole leaves in upper portion. After treatment with AIB $\left(3000 \mathrm{mg} \mathrm{L}^{-1}\right)$, the mini-cuttings were planted in tubes containing commercial substrate and kept in a greenhouse with controlled temperature and humidity. 120 days after planting we evaluated rooting, average number of roots/mini-cuttings, average length of roots, survival and mortality, callus formation, mini-cuttings with root and callus, emission of new shoots and leaf maintenance. The experiment was conducted in a completely randomized design, in a factorial scheme ( 2 clones x 5 doses of $\mathrm{N}$ ) with subplots divided in time, and variables submitted to ANOVA and Tukey. Factors influenced all variables analyzed, and clones responded differently to nitrogen doses in each harvesting season, indicating the importance of environmental and nutritional conditions in which the ministumps are maintained. The application of 104 to 380 $\mathrm{mg} \mathrm{L}^{-1}$ nitrogen in the nutrient solution is recommended.
\end{abstract}


Introdução

Ilex paraguariensis A.St.-Hil. é uma espécie arbórea nativa da Floresta Ombrófila Mista, de ocorrência natural na Argentina, Paraguai e em todos os estados do Sul do Brasil (Carvalho, 2003), região onde é considerada o principal produto florestal não madeireiro de grande importância econômica, social e ecológica (Duarte et al. 2019). Seus usos tradicionais são na forma de chimarrão (infusão quente) e tererê (infusão fria) e, mais recentemente, na forma de chá, infusão quente que se diferencia do chimarrão pelo beneficiamento dado às folhas. No cenário atual, a erva-mate tem se destacado com novos usos potenciais, como na produção de corantes, sorvetes, caramelos, medicamentos, perfumes, dentre outros (Dartora et al. 2013).

Apesar de sua importância econômica e crescente demanda, os plantios de erva-mate ainda são de origem seminal, com poucos critérios de seleção de plantas matrizes, fator que dificulta $o$ estabelecimento e uso de metodologias e padrões de manejo do plantio (Wendling e Santin 2015). A propagação vegetativa é uma alternativa viável para essa problemática e diversos estudos já indicam o potencial do uso dessas técnicas para propagação da espécie (Santin et al. 2015; Sturion et al. 2017; Duarte et al. 2019). A obtenção de mudas a partir de propagação vegetativa permite a seleção de materiais com características genéticas de interesse (Nascimento et al. 2018), uniformidade dos plantios, além da possibilidade de padronização dos tratos silviculturais e manejo, garantindo matéria-prima de qualidade para os mais variados fins.

A técnica de miniestaquia tem demonstrado resultados satisfatórios para a propagação de clones geneticamente superiores de espécies florestais e tem como principal vantagem a produtividade adequada de plantas com alto enraizamento, sem o uso de reguladores vegetais (Wendling et al. 2010). Apesar das vantagens, esta técnica apresenta maior sensibilidade às condições ambientais quando comparada com a estaquia convencional devido ao uso de material vegetativo mais herbáceo, requerendo assim cuidados na coleta $\mathrm{e}$ armazenamento dos propágulos e manutenção das condições ambientais de enraizamento, além de maior atenção às condições nutricionais, fitossanitárias e ambientais das minicepas (Goulart e Xavier 2010). Para erva-mate, a literatura demonstra que a miniestaquia é promissora, entretanto, o sucesso do enraizamento e qualidade das mudas pode ser muito dependente do material genético que se pretende propagar (Wendling et al. 2010; Kratz et al. 2015; Santin et al. 2015; Duarte et al. 2019).

A fertilização adequada das plantas a serem propagadas é um importante fator para o enraizamento e redução da mortalidade dos propágulos, sendo nitrogênio e potássio os nutrientes mais importantes para o crescimento vegetativo das plantas (Santin et al. 2008; Santin et al. 2013). O aumento da disponibilidade de nitrogênio promove maior síntese de citocininas nas plantas (Kojima et al. 2009), hormônio responsável pelo estímulo ao desenvolvimento de gemas laterais, novas brotações e folhas (Santin et al. 2008). Por outro lado, o excesso de $\mathrm{N}$ diminui a quantidade total de auxinas nas plantas (Kojima et al. 2009), hormônio responsável pelo desenvolvimento radicial, promoção da diferenciação celular e desenvolvimento de novas raízes. Não há registros de estudos que relacionem a influência da adubação nitrogenada de minicepas ao potencial rizogênico de miniestacas de erva-mate, sendo esse um gap a ser explorado na propagação vegetativa da espécie.

Tendo em vista o exposto e o potencial da miniestaquia na propagação em escala comercial, objetivou-se avaliar a influência de doses de nitrogênio, em solução nutritiva, na rizogênese de miniestacas de dois clones de Ilex paraguariensis, em diferentes épocas de coleta.

\section{Material e Métodos}

Os experimentos foram conduzidos entre os meses de abril de 2018 e agosto de 2019, nas dependências da Embrapa Florestas, em Colombo, Paraná. Como fonte de propágulos foram utilizadas brotações caulinares herbáceas de dois clones de erva-mate: EC22 e EC40. As minicepas dos dois clones foram mantidas em estufa, sob sistema semihidropônico tipo canaletão, com camada de drenagem de pedra brita, cobertas por camadas de areia lavada, esta última em contato direto com as minicepas. O manejo das canaletas consistiu em limpeza semanal e lavagem mensal com água corrente. Os clones são provenientes de plantas propagadas via estaquia convencional, oriundas de duas matrizes de um teste de procedências e progênies com 22 anos, instalado no município de Ivaí, Paraná $\left(25^{\circ} 01^{\prime} \mathrm{S}, 50^{\circ} 47^{\prime} \mathrm{W}\right.$ e $650-750 \mathrm{~m}$ altitude)

As minicepas receberam diariamente solução de fertirrigação com cinco concentrações de nitrogênio (N) (Tabela 1). Os demais elementos constituintes das soluções foram aplicados igualmente para todos os tratamentos, sendo: $25 \mathrm{mg}$ $\mathrm{L}^{-1}$ de Fósforo, $200 \mathrm{mg} \mathrm{L}^{-1}$ de Potássio, $200 \mathrm{mg} \mathrm{L}^{-1}$ de Cálcio, $45 \mathrm{mg} \mathrm{L}^{-1}$ de Magnésio, 76,9 $\mathrm{mg} \mathrm{L}^{-1}$ de Enxofre, 1,5 $\mathrm{mg} \mathrm{L}^{-1}$ de Boro, 0,10 $\mathrm{mg} \mathrm{L}^{-1}$ de Cobre, $5 \mathrm{mg} \mathrm{L}^{-1}$ de Ferro, $1 \mathrm{mg} \mathrm{L}^{-1}$ de Manganês, 0,7 mg L${ }^{1}$ de Zinco e $0,07 \mathrm{mg} \mathrm{L}^{-1}$ de Molibdênio. As soluções foram distribuídas via gotejamento, três vezes ao dia, em um total diário de $5 \mathrm{~L} \mathrm{~m}^{-2}$. Além da irrigação via gotejamento, as minicepas receberam irrigação via aspersão três vezes ao dia, em um total diário de aproximadamente $3,75 \mathrm{~L} \mathrm{~m}^{-2}$. 


\section{Duarte et al.}

Tabela 1. Nitrogênio total $(\mathrm{N})$, Nitrato $\left(\mathrm{NO}_{3}\right)$ e Amônia $\left(\mathrm{NH}_{4}\right)$, potencial hidrogeniônico $(\mathrm{pH})$ e condutividade elétrica (CE) das soluções utilizadas na fertirrigação de minicepas de Ilex paraguariensis.

\begin{tabular}{cccccc} 
Tratamento & $\begin{array}{c}\text { Dose de N total } \\
\left(\mathrm{mg} \mathrm{L}^{-1}\right)\end{array}$ & $\begin{array}{c}\mathrm{NO}_{3} \\
\left(\mathrm{mg} \mathrm{L}^{-1}\right)\end{array}$ & $\begin{array}{c}\mathrm{NH}_{4} \\
\left(\mathrm{mg} \mathrm{L}^{-1}\right)\end{array}$ & $\mathrm{pH}$ & $\begin{array}{c}\mathrm{CE} \\
\left(\mathrm{dS} \mathrm{cm}^{-1}\right)\end{array}$ \\
\hline 1 & 104 & 54,0 & 50,0 & 5,0 & 2,21 \\
$2^{*}$ & 206 & 156,0 & 50,0 & 5,0 & 2,47 \\
3 & 380 & 143,0 & 137,0 & 5,0 & 3,00 \\
4 & 760 & 433,0 & 327,0 & 5,0 & 3,76 \\
5 & 1142 & 624,0 & 518,0 & 5,0 & 4,74 \\
\hline
\end{tabular}

*Dose padrão para miniestaquia de erva-mate (Wendling e Santin 2015).

As brotações foram coletadas em quatro épocas do ano, de acordo com a disponibilidade de propágulos, nas datas: 23/04/18 (outono), 27/06/18 (outono), 25/02/19 (verão) e 25/04/19 (outono). As coletas foram realizadas nas primeiras horas do dia, sendo as miniestacas confeccionadas utilizando-se todas as porções das brotações, com $8 \pm 1 \mathrm{~cm}$, mantendo de 2 a 3 folhas inteiras na porção superior. Para a manutenção da hidratação as miniestacas foram mantidas em água até o momento do plantio.

As bases das miniestacas foram tratadas com solução $50 \%$ hidroalcoólica de ácido indol-3butírico (AIB) na concentração de $3000 \mathrm{mg} \mathrm{L}^{-1}$, por 10 segundos (Brondani et al. 2008), e então plantadas em tubetes de polipropileno de $53 \mathrm{~cm}^{3}$, contendo substrato comercial, composto por turfa de Sphagnum, vermiculita expandida e casca de arroz carbonizada (condutividade elétrica: $0,6 \mathrm{mS} \mathrm{cm} \mathrm{cm}^{-1}$; capacidade de retenção de água: 140 \%; pH 5,0). As miniestacas foram mantidas em casa de vegetação com nebulização intermitente por 15 segundos a cada 6 a 10 minutos $(80 \%$ de umidade relativa e temperatura entre 20 e $30^{\circ} \mathrm{C}$ ).

Transcorridos 120 dias em casa de vegetação, foram avaliadas em todas as miniestacas dos tratamentos: a porcentagem de enraizamento, número médio de raízes por miniestaca, comprimento médio das três maiores raízes por miniestaca $(\mathrm{cm})$, porcentagem de miniestacas vivas (sem raízes e calos, mas permaneceram vivas), porcentagem de miniestacas mortas (com tecidos necrosados), porcentagem de miniestacas com formação de calos, porcentagem de miniestacas com formação de calos e raízes simultaneamente, porcentagem de miniestacas com emissão de novas brotações (de pelo menos $2 \mathrm{~mm}$ de comprimento) e manutenção de folhas originais nas miniestacas.

Os tratamentos consistiram em cinco repetições, sendo que o número de miniestacas por tratamento variou entre 0 e 15 em função da produtividade de brotações das minicepas. O delineamento experimental foi inteiramente casualizado, em esquema fatorial 5 x 2 (cinco doses de nitrogênio $x$ dois clones) e em parcelas subdivididas (quatro épocas de coleta). As variáveis foram avaliadas quanto à homogeneidade das variâncias e normalidade dos resíduos e posteriormente submetidas à Análise de Variância
Desbalanceada. As médias foram comparadas pelo teste de Tukey, ao nível de $5 \%$ de probabilidade.

\section{Resultados}

A análise de variância demonstrou interação entre os fatores doses de $\mathrm{N}$ e clones para todas as variáveis analisadas e diferenças significativas entre os tratamentos. As minicepas do clone EC22 não produziram brotações suficientes para a produção de miniestacas nas duas primeiras coletas, quando submetidas à maior dose de $\mathrm{N}\left(1142 \mathrm{mg} \mathrm{L}^{-1}\right)$. A relação de interação entre os fatores e os valores obtidos para as variáveis foram distintos em cada coleta e dose de nitrogênio. As médias de porcentagem de miniestacas enraizadas estão apresentadas na Figura 1.

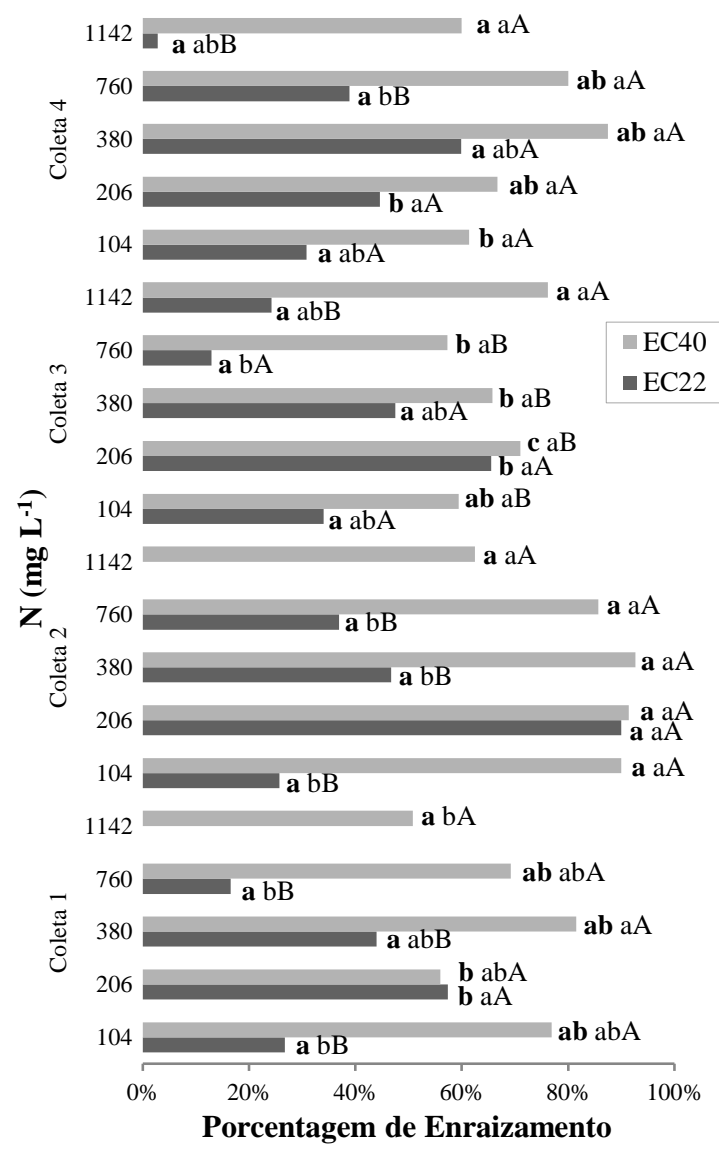

Figura 1. Porcentagem de enraizamento aos 120 dias após plantio de miniestacas de dois clones de Ilex 
paraguariensis produzidos em cinco doses de nitrogênio e quatro épocas de coleta. Letras em negrito para as épocas de coleta, letras minúsculas para as doses de nitrogênio e letras maiúsculas para os clones. Médias seguidas da mesma letra não diferem estatisticamente entre si pelo teste de Tukey ao nível de $5 \%$ de probabilidade $(\mathrm{p}<0,05)$.

Com relação à porcentagem de enraizamento, os clones responderam de maneira diferente às doses de $\mathrm{N}$ e o clone EC40 apresentou valor superior em relação ao clone EC22 em todos os tratamentos, exceto na coleta 1 para dose $206 \mathrm{mg} \mathrm{L}^{-1}$. O clone EC40 apresentou mais de $90 \%$ de enraizamento nas doses 104, 206 e $380 \mathrm{mg} \mathrm{L}^{-1}$ de $\mathrm{N}$ da coleta 2, sendo estes os maiores valores observados. O clone EC22 apresentou $90 \%$ de enraizamento na coleta 2 , quando submetido a $206 \mathrm{mg} \mathrm{L}^{-1}$ de $\mathrm{N}$, sendo a maior porcentagem de enraizamento obtida para este clone.
Nos demais tratamentos o enraizamento das miniestacas do clone EC22 permaneceu abaixo de $65 \%$.

Para as variáveis número médio de raízes e comprimento médio de raízes houve diferença significativa entre as coletas para ambos os clones. $\mathrm{O}$ clone EC40 apresentou número e comprimento médio de raízes superior ou semelhante ao clone EC22 em todos os tratamentos. De maneira geral, para EC40 as doses 760 e 1142 mg L L $^{-1}$ proporcionaram as menores médias de número e comprimento de raízes. Para EC22 as doses 206, 380 e $760 \mathrm{mg} \mathrm{L}^{-1}$ resultaram em maiores médias de número de raízes nas coletas 1 e 2 , não havendo diferenças significativas para as doses nas demais coletas. O comprimento de raízes foi favorecido pelas doses 206, 380 e $760 \mathrm{mg} \mathrm{L}^{-1}$ nas coletas 1,2 e 3 (Tabela 2).

Tabela 2. Número médio de raízes por miniestaca e comprimento médio $(\mathrm{cm})$ das três maiores raízes por miniestaca em dois clones de Ilex paraguariensis produzidos em cinco doses de nitrogênio e quatro épocas de coleta, aos 120 dias após plantio.

\begin{tabular}{|c|c|c|c|c|c|c|c|c|}
\hline \multirow{2}{*}{$\begin{array}{c}\mathrm{N} \\
\left(\mathrm{mg} \mathrm{L}^{-1}\right)\end{array}$} & \multicolumn{2}{|c|}{ Coleta 1} & \multicolumn{2}{|c|}{ Coleta 2} & \multicolumn{2}{|c|}{ Coleta 3} & \multicolumn{2}{|c|}{ Coleta 4} \\
\hline & EC 22 & EC 40 & EC 22 & EC 40 & EC 22 & EC 40 & EC 22 & EC 40 \\
\hline \multicolumn{9}{|c|}{ Número médio de raízes } \\
\hline 104 & $2.1 \mathrm{~b} \mathrm{bB}$ & 10.4 a aA & $2.2 \mathrm{~b} \mathrm{bB}$ & $10.1 \mathrm{a} a \mathrm{~A}$ & $8.1 \mathrm{a} \mathrm{aB}$ & $12.5 \mathrm{a} a A$ & $3.1 \mathrm{ab} \mathrm{aA}$ & $4.6 \mathrm{~b} \mathrm{aA}$ \\
\hline 206 & $10.1 \mathrm{a} a \mathrm{~A}$ & $10.1 \mathrm{a} a \mathrm{~A}$ & $10.1 \mathrm{a} \mathrm{aA}$ & $10.3 \mathbf{a} \mathrm{aA}$ & $8.3 \mathbf{a} \mathrm{aA}$ & $10.6 \mathbf{a} \mathrm{abA}$ & $3.7 \mathrm{~b}$ aA & $4.6 \mathrm{~b} \mathrm{aA}$ \\
\hline 380 & $5.7 \mathbf{a b} a b B$ & 10.9 a aA & $4.2 \mathbf{a} \mathrm{bB}$ & $11.0 \mathbf{a} \mathrm{aA}$ & $4.1 \mathbf{a} \mathrm{aB}$ & $10.6 \mathbf{a} \mathrm{abA}$ & $4.7 \mathbf{a} \mathrm{aB}$ & $8.2 \mathbf{a}$ aA \\
\hline 760 & $4,9 \mathbf{a b} \mathrm{bA}$ & $7.6 \mathbf{a b}$ abA & $10.2 \mathbf{a} \mathrm{aA}$ & $8.5 \mathbf{a b}$ aA & $4.7 \mathbf{a b} \mathrm{aB}$ & $11.2 \mathrm{a} \mathrm{abA}$ & $2.9 \mathrm{~b}$ aA & $5.5 \mathrm{~b}$ aA \\
\hline 1142 & - & $6.4 \mathbf{a} \mathrm{bA}$ & - & $6.7 \mathbf{a} a A$ & $1.9 \mathbf{a} \mathrm{aA}$ & $7.3 \mathbf{a} \mathrm{bA}$ & $0.4 \mathbf{a} \mathrm{aA}$ & 3.9 a aA \\
\hline \multicolumn{9}{|c|}{ Comprimento médio das três maiores raízes $(\mathrm{cm})$} \\
\hline 104 & $2.9 \mathbf{a b} \mathrm{abA}$ & $4.0 \mathbf{a} \mathrm{aA}$ & $1.8 \mathrm{~b} \mathrm{bB}$ & $4.9 \mathrm{a}$ aA & 4.7 a aA & $4.3 \mathbf{a} \mathrm{aA}$ & 2.8 ab aA & $2.0 \mathrm{~b}$ aA \\
\hline 206 & $4.3 \mathbf{a} \mathrm{aA}$ & $3.3 \mathbf{a b}$ abA & $4.9 \mathbf{a} \mathrm{aA}$ & $4.3 \mathbf{a} \mathrm{aA}$ & $3.5 \mathbf{a b} \mathrm{aA}$ & $3.6 \mathbf{a b}$ aA & $2.2 \mathbf{b}$ abA & $1.8 \mathrm{~b}$ aA \\
\hline 380 & $3.2 \mathbf{a} \mathrm{abA}$ & $2.1 \mathrm{~b}$ abA & $1.8 \mathbf{a ~ b B}$ & $4.7 \mathbf{a}$ aA & 2.9 a aA & $2.7 \mathbf{b}$ aA & $3.7 \mathbf{a} \mathrm{aA}$ & $2.7 \mathbf{b}$ aA \\
\hline 760 & $0.6 \mathbf{a} \mathrm{bA}$ & $1.6 \mathbf{a} \mathrm{bA}$ & $1.9 \mathrm{a} \mathrm{bA}$ & $3.5 \mathrm{a}$ aA & $2.8 \mathbf{a} \mathrm{aA}$ & $3.1 \mathrm{a}$ aA & $2.2 \mathbf{a} \mathrm{abA}$ & $1.9 \mathbf{a} \mathrm{aA}$ \\
\hline 1142 & - & $1.3 \mathbf{a b} \mathrm{bA}$ & - & $3.6 \mathrm{a} a \mathrm{~A}$ & $0.8 \mathbf{a} \mathrm{aA}$ & $2.6 \mathbf{a b} \mathrm{aA}$ & $0.2 \mathrm{a} \mathrm{bA}$ & $1.0 \mathrm{~b} \mathrm{aA}$ \\
\hline
\end{tabular}

Letras em negrito para as épocas de coleta, letras minúsculas para as doses de nitrogênio e letras maiúsculas para os clones. Médias seguidas das mesmas letras não diferem estatisticamente entre si pelo teste de Tukey ao nível de $5 \%$ de probabilidade $(\mathrm{p}<0.05)$.

A porcentagem de miniestacas vivas não apresentou diferença entre as coletas para EC40, sendo que as demais variáveis diferiram entre as coletas para ambos os clones (Tabela 3). As maiores médias de miniestacas vivas foram observadas para o clone EC22 na dose $760 \mathrm{mg} \mathrm{L}^{-1}$ de $\mathrm{N}$ (coletas $1 \mathrm{e}$ 3) e na dose $104 \mathrm{mg} \mathrm{L}^{-1}$ de N (coleta 2). Nas coletas 1 e 3 foi possível observar as maiores médias de miniestacas mortas, para os dois clones. De maneira geral, o clone EC22 apresentou maiores porcentagens de miniestacas com calo, em todas as coletas e doses, quando comparado com EC40. As doses 206 (coletas 1, 2 e 3), 380 (coleta 4) e $760 \mathrm{mg}$ $\mathrm{L}^{-1}$ de $\mathrm{N}$ (coleta 3 ) apresentaram as menores médias de calos para EC 22. Para EC40, a dose $1142 \mathrm{mg} \mathrm{L}^{-1}$ de $\mathrm{N}$ na coleta 1 apresentou a maior porcentagem de miniestacas com calos, e na coleta 4 somente a dose $380 \mathrm{mg} \mathrm{L}^{-1}$ de $\mathrm{N}$ diferiu das demais, com menor média para essa variável. Em todos os tratamentos e coletas foram observadas miniestacas com a presença simultânea de calos e raízes. 
Tabela 3. Porcentagem de miniestacas vivas, mortalidade, com formação de calos e com presença de calo e raiz, em dois clones Ilex paraguariensis produzidos em cinco doses de nitrogênio e quatro épocas de coleta, aos 120 dias após plantio.

\begin{tabular}{|c|c|c|c|c|c|c|c|c|}
\hline \multirow{2}{*}{$\begin{array}{c}\mathrm{N} \\
\left(\mathrm{mg} \mathrm{L}^{-1}\right)\end{array}$} & \multicolumn{2}{|c|}{ Coleta 1} & \multicolumn{2}{|c|}{ Coleta 2} & \multicolumn{2}{|c|}{ Coleta 3} & \multicolumn{2}{|c|}{ Coleta 4} \\
\hline & $\mathrm{EC} 22$ & EC40 & $\mathrm{EC} 22$ & EC40 & EC22 & EC40 & $\mathrm{EC} 22$ & $\mathrm{EC} 40$ \\
\hline \multicolumn{9}{|c|}{ Miniestacas vivas (\%) } \\
\hline 104 & $0.0 \mathrm{~b} b A$ & $0.0 \mathrm{a} \mathrm{aA}$ & $14.3 \mathbf{a}$ aA & $6.7 \mathbf{a}$ aA & $1.2 \mathbf{a b} \mathrm{abA}$ & $8.0 \mathrm{a} \mathrm{aA}$ & 6.2 ab aA & $2.9 \mathbf{a} \mathrm{aA}$ \\
\hline 206 & $2.0 \mathrm{a} b \mathrm{~b}$ & $0.0 \mathbf{a}$ aA & $6.7 \mathbf{a} \mathrm{aA}$ & $0.0 \mathbf{a} \mathrm{aA}$ & $0.0 \mathrm{a} b \mathrm{~b}$ & $0.0 \mathrm{a} \mathrm{aA}$ & $0.0 \mathbf{a} \mathrm{aA}$ & $0.0 \mathbf{a}$ aA \\
\hline 380 & $0.0 \mathrm{a} b \mathrm{~b}$ & $0.0 \mathbf{a} \mathrm{aA}$ & 8.9 a aA & $0.0 \mathrm{a} a \mathrm{~B}$ & $5.0 \mathbf{a} \mathrm{abA}$ & $4.0 \mathbf{a} \mathrm{aA}$ & $0.0 \mathrm{a} \mathrm{aA}$ & $1.3 \mathbf{a} \mathrm{aA}$ \\
\hline 760 & $15.9 \mathbf{a} \mathrm{aA}$ & $1.5 \mathrm{a} \mathrm{aB}$ & $6.9 \mathbf{a b} \mathrm{aA}$ & $2.9 \mathbf{a} \mathrm{aA}$ & $18.6 \mathbf{a} \mathrm{aA}$ & $1.3 \mathbf{a ~ a B}$ & $2.0 \mathrm{~b}$ aA & $0.0 \mathbf{a} \mathrm{aA}$ \\
\hline 1142 & - & $0.0 \mathbf{a}$ aA & - & $6.3 \mathbf{a}$ aA & $0.0 \mathrm{a} \mathrm{bA}$ & $0.0 \mathrm{a} \mathrm{aA}$ & 5.7 a aA & $0.0 \mathbf{a}$ aA \\
\hline \multicolumn{9}{|c|}{ Mortalidade (\%) } \\
\hline 104 & 23.3 a aA & $17.1 \mathbf{a b} \mathrm{bA}$ & $8.6 \mathrm{~b} \mathrm{aA}$ & $0.0 \mathrm{~b}$ bA & 36.9 a abA & $32.6 \mathbf{a} \mathrm{aA}$ & $0.0 \mathrm{~b}$ aA & $2.9 \mathrm{~b}$ aA \\
\hline 206 & $19.3 \mathbf{a} a B$ & $40.0 \mathbf{a} \mathrm{aA}$ & $0.0 \mathrm{~b}$ aA & $2.9 \mathrm{c} \mathrm{bA}$ & $23.5 \mathrm{a}$ bA & $24.0 \mathbf{a b}$ aA & $0.0 \mathrm{~b}$ aA & $12.2 \mathbf{b}$ aA \\
\hline 380 & 19.9 ab aA & $11.8 \mathbf{a b} \mathrm{bA}$ & $4.4 \mathrm{~b}$ aA & $5.5 \mathrm{~b}$ bA & $30.0 \mathbf{a}$ abA & $26.2 \mathbf{a} \mathrm{aA}$ & $3.1 \mathrm{~b}$ aA & $6.2 \mathrm{~b}$ aA \\
\hline 760 & $23.8 \mathrm{~b}$ aA & $16.9 \mathrm{~b} b \mathrm{bA}$ & $0.0 \mathrm{c} \mathrm{aA}$ & $2.9 \mathrm{~b}$ bA & $68.1 \mathbf{a} \mathrm{aA}$ & $37.3 \mathbf{a} \mathrm{aB}$ & $10.0 \mathrm{~b}$ aA & $3.3 \mathrm{~b}$ aA \\
\hline 1142 & - & $12.5 \mathrm{a} \mathrm{bA}$ & - & $31.3 \mathbf{a}$ aA & $26.1 \mathrm{a} b A$ & 19.1 a aA & $0.0 \mathrm{~b}$ aA & $7.1 \mathrm{~b}$ aA \\
\hline \multicolumn{9}{|c|}{ Miniestacas com calos (\%) } \\
\hline 104 & 49.9 ab aA & 5.9 b bB & $51.4 \mathbf{a b}$ aA & $3.3 \mathrm{~b}$ aB & $32.2 \mathbf{b}$ abA & $0.0 \mathrm{~b}$ aB & $63.1 \mathrm{a} \mathrm{bA}$ & $32.9 \mathbf{a} \mathrm{aB}$ \\
\hline 206 & $21.3 \mathbf{b}$ bA & $4.0 \mathrm{~b} b B$ & $3.3 \mathrm{~b}$ bA & $5.7 \mathrm{~b}$ aA & $11.0 \mathrm{~b} \mathrm{bA}$ & $5.0 \mathrm{~b}$ aA & $55.4 \mathrm{a}$ bcA & $21.1 \mathrm{a} a b B$ \\
\hline 380 & 35.9 a abA & $6.6 \mathbf{a ~ b B}$ & 39.9 a abA & $1.8 \mathrm{a} \mathrm{aB}$ & $17.5 \mathrm{~b}$ abA & $4.0 \mathbf{a} \mathrm{aA}$ & 36.9 a cA & $5.0 \mathrm{a} b B$ \\
\hline 760 & $43.8 \mathbf{a b}$ abA & $12.3 \mathrm{a} \mathrm{bB}$ & 56.9 a aA & $8.6 \mathrm{a} a \mathrm{~B}$ & $0.0 \mathrm{c} \mathrm{bA}$ & $4.0 \mathrm{~b}$ aA & 49.1 a bcA & $16.7 \mathbf{a} \mathrm{abB}$ \\
\hline 1142 & - & $36.7 \mathbf{a}$ aA & - & $0.0 \mathrm{~b}$ aA & $50.3 \mathbf{b}$ aA & $4.8 \mathrm{~b}$ aB & $91.4 \mathbf{a}$ aA & $32.9 \mathbf{a} \mathrm{aB}$ \\
\hline \multicolumn{9}{|c|}{ Miniestacas com raiz e calo $(\%)$} \\
\hline 104 & $23.3 \mathbf{a}$ abB & 75.5 ab aA & 25.7 a bB & 90.0 a aA & $34.0 \mathbf{a} \mathrm{abA}$ & $59.4 \mathrm{~b}$ aA & 20.0 a bcB & $61.4 \mathbf{a b}$ aA \\
\hline 206 & $51.8 \mathrm{~b}$ aA & $54.7 \mathbf{b}$ abA & $90.0 \mathbf{a}$ aA & $91.4 \mathbf{a}$ aA & $63.0 \mathbf{a b}$ aA & $71.0 \mathbf{a b}$ aA & $43.1 \mathrm{~b}$ abB & 66.7 ab aA \\
\hline 380 & $44.0 \mathrm{a} a b B$ & $81.5 \mathbf{a b}$ aA & $44.4 \mathbf{a}$ bB & 92.7 a aA & 30.0 a $a b B$ & $61.5 \mathrm{~b}$ aA & $58.5 \mathrm{a}$ aA & $72.5 \mathbf{a b}$ aA \\
\hline 760 & $16.5 \mathrm{a} b \mathrm{bB}$ & $66.1 \mathbf{a b}$ abA & 29.8 a bB & 85.7 a aA & 0.9 a bB & 54.7 b aA & $36.9 \mathbf{a} \mathrm{abB}$ & 80.0 ab aA \\
\hline 1142 & - & 40.8 a bA & - & $62.5 \mathrm{a} \mathrm{bA}$ & $17.8 \mathbf{a} \mathrm{bA}$ & 42.8 a aA & $2.8 \mathrm{~b} \mathrm{cB}$ & $57.1 \mathrm{a}$ aA \\
\hline
\end{tabular}

Letras em negrito para as épocas de coleta, letras minúsculas para as doses de nitrogênio e letras maiúsculas para os clones. Médias seguidas das mesmas letras não diferem estatisticamente entre si pelo teste de Tukey ao nível de $5 \%$ de probabilidade $(\mathrm{p}<0.05)$.

A emissão de novas brotações nas miniestacas foi, de maneira geral, superior no clone EC40, exceto na coleta 3, dose $380 \mathrm{mg} \mathrm{L}^{-1}$ de N, em que o clone EC22 foi superior. As maiores porcentagens de miniestacas com brotos foram observadas na coleta 3 , principalmente naquelas do clone EC40. A manutenção de folhas foi similar entre os clones, havendo diferença significativa apenas na dose $760 \mathrm{mg} \mathrm{L}^{-1}$ de $\mathrm{N}$, na coleta 4. De maneira geral, as doses de nitrogênio não influenciaram significativamente essa variável, havendo diferenças entre as doses apenas nas coletas 1 e 2 (Tabela 4).

Tabela 4. Porcentagem de miniestacas com emissão de novas brotações e manutenção de folhas originais, em dois clones Ilex paraguariensis produzidos em cinco doses de nitrogênio e quatro épocas de coleta, aos 120 dias após plantio.

\begin{tabular}{|c|c|c|c|c|c|c|c|c|}
\hline \multirow{2}{*}{$\begin{array}{c}\mathrm{N} \\
\left(\mathrm{mg} \mathrm{L}^{-1}\right)\end{array}$} & \multicolumn{2}{|c|}{ Coleta 1} & \multicolumn{2}{|c|}{ Coleta 2} & \multicolumn{2}{|c|}{ Coleta 3} & \multicolumn{2}{|c|}{ Coleta 4} \\
\hline & EC 22 & EC 40 & EC 22 & EC 40 & EC 22 & EC 40 & EC 22 & EC 40 \\
\hline \multicolumn{9}{|c|}{ Emissão de novas brotações (\%) } \\
\hline 104 & $0.0 \mathrm{a} a \mathrm{aB}$ & $21.7 \mathbf{a}$ aA & $5.7 \mathbf{a} a A$ & $13.3 \mathbf{a} \mathrm{aA}$ & $0.0 \mathbf{a} b B$ & $22.9 \mathbf{a} \mathrm{abA}$ & $1.5 \mathrm{a} a \mathrm{~B}$ & $12.9 \mathbf{a} \mathrm{aA}$ \\
\hline 206 & $0.0 \mathrm{~b}$ aA & $2.7 \mathrm{~b} b A$ & $13.4 \mathbf{a} \mathrm{aA}$ & $5.7 \mathrm{~b}$ aA & $15.5 \mathrm{a} a b B$ & $28.5 \mathbf{a} \mathrm{abA}$ & $3.1 \mathrm{~b}$ aA & $8.9 \mathrm{~b}$ aA \\
\hline 380 & $2.2 \mathrm{~b}$ aA & $3.8 \mathrm{~b}$ bA & $0.0 \mathrm{~b}$ aB & $12.7 \mathbf{a} \mathrm{aA}$ & $27.5 \mathbf{a} \mathrm{aA}$ & $16.0 \mathbf{a} \mathrm{bB}$ & $6.1 \mathrm{~b}$ aA & $10.0 \mathbf{a} \mathrm{aA}$ \\
\hline 760 & $0.0 \mathbf{a} \mathrm{aA}$ & $1.5 \mathrm{~b}$ bA & $0.0 \mathbf{a} \mathrm{aA}$ & $8.6 \mathbf{a b}$ aA & $0.0 \mathbf{a} b B$ & $18.7 \mathbf{a} \mathrm{bA}$ & $0.0 \mathbf{a} \mathrm{aA}$ & $9.9 \mathbf{a b}$ aA \\
\hline 1142 & - & $3.3 \mathbf{b}$ bA & - & $6.3 \mathrm{~b}$ aA & $0.6 \mathbf{a} b B$ & $38.1 \mathbf{a}$ aA & $0.0 \mathbf{a} \mathrm{aA}$ & $2.9 \mathrm{~b}$ aA \\
\hline \multicolumn{9}{|c|}{ Manutenção de folhas originais (\%) } \\
\hline 104 & 76.7 a aA & $78.3 \mathbf{a b}$ aA & $91.4 \mathbf{a} \mathrm{aA}$ & 96.7 a aA & $59.3 \mathbf{a}$ aA & $56.6 \mathrm{~b}$ aA & 98.5 a aA & 95.7 a aA \\
\hline 206 & 74.9 a aA & $53.3 \mathrm{~b}$ aA & 96.7 a aA & $94.3 \mathbf{a}$ abA & $72.5 \mathbf{a}$ aA & $73.5 \mathbf{a b}$ aA & $100.0 \mathbf{a} \mathrm{aA}$ & $85.6 \mathbf{a}$ aA \\
\hline 380 & $75.8 \mathbf{a b}$ aA & 79.9 a aA & $93.3 \mathbf{a b} \mathrm{aA}$ & 89.1 a abA & $62.5 \mathrm{~b}$ aA & $63.8 \mathbf{a} \mathrm{aA}$ & 96.9 a aA & $77.5 \mathbf{a} \mathrm{aA}$ \\
\hline 760 & 31.4 b bA & $50.8 \mathbf{b}$ aA & $100.0 \mathrm{a}$ aA & $91.4 \mathbf{a} \mathrm{abA}$ & $32.6 \mathbf{b}$ aA & $52.0 \mathrm{~b}$ aA & $68.0 \mathbf{a b} \mathrm{aB}$ & $93.3 \mathbf{a}$ aA \\
\hline 1142 & - & $80.8 \mathbf{a}$ aA & - & $50.0 \mathrm{a} \mathrm{bA}$ & $75.0 \mathbf{a}$ aA & $71.4 \mathbf{a}$ aA & $100.0 \mathbf{a}$ aA & 85.7 a aA \\
\hline
\end{tabular}

Letras em negrito para as épocas de coleta, letras minúsculas para as doses de nitrogênio e letras maiúsculas para os clones. Médias seguidas das mesmas letras não diferem estatisticamente entre si pelo teste de Tukey ao nível de $5 \%$ de probabilidade $(\mathrm{p}<0.05)$. 


\section{Discussão}

Os resultados obtidos neste estudo evidenciam o potencial da miniestaquia na propagação de erva-mate, como já observado por outros autores ao utilizar materiais da espécie com diferentes idades, aplicação de reguladores vegetais e diferentes tamanhos de propágulos (Kratz et al. 2015; Stuepp et al. 2015; Stuepp et al. 2017; Duarte et al. 2019). Esses resultados refletem a adaptabilidade do material às condições ambientais e nutricionais às quais foram submetidas, evidenciando também a manutenção do vigor das minicepas no período de realização do experimento (Stuepp et al., 2017). As médias de porcentagem de enraizamento obtidas no presente trabalho corroboram com as observadas por Brondani et al. (2008), as quais variaram de 62,5 a 44,6\%, aos 150 dias e por Sá et al. (2018), com variação na taxa de enraizamento de 68,9 a 27,3\%. Ainda, os resultados são considerados satisfatórios para a espécie e podem estar relacionados a juvenilidade do material propagado (Wendling et al. 2010)

$\mathrm{O}$ genótipo, a dose de $\mathrm{N}$ e a época de coleta exerceram influência no enraizamento, podendo-se afirmar que o sucesso da miniestaquia está aliada a um conjunto de fatores. $\mathrm{O}$ material genético apresentou influência direta no enraizamento das miniestacas, sendo a capacidade rizogênica do clone EC40 superior ao EC22. A influência do material genético, sobretudo para o enraizamento de plantas, é um dos desafios no estabelecimento de plantios clonais de erva-mate e, de acordo com Nascimento et al. (2018), a época de coleta dos propágulos exerce influência no enraizamento, havendo diferenças significativas entre materiais genéticos, ainda que da mesma espécie. Com relação a épocas de coleta, Stuepp et al. (2017) observaram menores taxas de enraizamento em estacas de erva-mate coletadas no período do verão, sendo a mesma tendência observada para o clone EC40. Sá et al. (2018), por sua vez, observaram melhor enraizamento em miniestacas coletadas na primavera, com média de $68,9 \%$ de enraizamento.

O nitrogênio está ligado, de forma direta ou indireta, à todas as rotas metabólicas vegetais devido à sua participação na atividade fotossintética, promovendo assim maior acúmulo de biomassa (Taiz et al. 2017). Além disso, estudos indicam que o nitrogênio tem influência direta no índice de produtividade de minicepas e no acúmulo de matéria seca nas mudas produzidas (Rocha et al. 2015). Apesar dessas variáveis não terem sido mensuradas, foi possível perceber uma tendência na redução da disponibilidade de propágulos à medida que as doses de $\mathrm{N}$ aumentavam, para ambos os clones. Assim, apesar de sua importância em diversos aspectos do crescimento e desenvolvimento vegetal, é prudente considerar um ponto crítico a partir do qual a planta passa a responder de maneira indesejada à disponibilidade de N, como observado nos tratamentos em que as minicepas foram submetidas a 760 ou $1142 \mathrm{mg} \mathrm{L}^{-1}$ de $\mathrm{N}$.

Hartmann et al. (2017) afirmam que o $\mathrm{N}$ possui relação inversa com o enraizamento de miniestacas, em função da redução da relação $\mathrm{C} / \mathrm{N}$, sendo que tanto o excesso quanto o déficit de $\mathrm{N}$ são desfavoráveis à rizogênese, principalmente por afetarem a síntese de ácidos nucléicos e proteínas. Neste experimento, esse fato pode ser observado pela redução da porcentagem de miniestacas enraizadas nas maiores doses de $\mathrm{N}$, entretanto, a menor dose utilizada não foi suficiente para observar efeito semelhante. Efeito benéfico do $\mathrm{N}$ foi discutido por Rocha et al. (2015), os quais afirmam que para clones de Eucalyptus, doses adequadas deste nutriente podem reduzir o tempo de enraizamento das miniestacas. Os autores ainda destacam que além da nutrição, a presença de carboidratos e outros metabólitos, bem como o material genético e o grau de juvenilidade dos propágulos são fatores preponderantes para formação de raízes.

Os resultados observados durante o processo de enraizamento permitem afirmar que a rizogênese da erva-mate acontece tanto de maneira direta como indireta. Duarte et al. (2019) relataram que a formação de calos não é determinante para a formação de raízes adventícias no caso de $I$. paraguariensis, podendo essas serem originadas de células cambiais ou a partir de calos (Hartmann et al. 2017). Pode-se destacar ainda a diferença de formação de calos entre os dois clones estudados, sendo que EC22 apresentou maiores porcentagens em relação a EC40. Assim, observa-se que o processo de calogênese foi intrínseco ao material genético, conforme já observado por Stuepp et al. (2013).

A emissão de novos brotos foi maior nas miniestacas provenientes do clone EC40 e, quando houve influência das doses de $\mathrm{N}$, as maiores médias foram observadas nos tratamentos com as menores doses deste nutriente. As porcentagens de brotações de ambos os clones foram similares às observadas por Tronco et al. (2015) ao avaliar microestacas de erva-mate. As porcentagens abaixo de $30 \%$ para novas brotações podem estar relacionadas à disponibilidade energética das miniestacas, priorizando o uso de carboidratos no processo de rizogênese em detrimento da produção de brotos. Somkuwar et al. (2011) reportam que a presença de gemas axilares, o uso de reguladores vegetais, temperatura, condições fitossanitárias das matrizes e a disponibilidade de carboidratos também são fatores relacionados à indução de brotações, estando este último intimamente relacionado a disponibilidade de $\mathrm{N}$ nas plantas.

A manutenção de folhas, apesar de não ser um parâmetro atrelado ao sucesso do processo de propagação vegetativa, é uma importante fonte de energia e síntese de substâncias relacionadas à rizogênese (Xavier et al. 2013). Taiz et al. (2017) 
sugerem que a biossíntese do ácido indol-3-acético (AIA) está diretamente associada à tecidos com rápido crescimento celular, tais como folhas jovens. Este fitohormônio está ligado também à promoção do crescimento radicial e tal relação fica evidente na observação do clone EC40 na coleta 3, onde as miniestacas apresentaram menores porcentagens de enraizamento e de manutenção de folhas. A relação entre essas duas variáveis também foi observada por Wendling et al. (2010) em miniestacas de Liquidambar styraciflua, e destacam a importância de estudos visando esclarecer a relação entre o conteúdo de AIA nas folhas e o desenvolvimento do sistema radicial de plantas propagadas vegetativamente.

Estudos conduzidos em campo destacam a importância da disponibilidade de $\mathrm{N}$ para a qualidade das mudas e produtividade da erva-mate (Santin et al. 2008; Santin et al. 2013; Santin et al. 2015; Barbosa et al. 2018), entretanto, não estabelecem padrões adequados de nutrição da espécie quando mantida em sistema de minijardim clonal, em que a exportação deste nutriente também é alta. Os resultados obtidos neste estudo indicam que há limites para a disponibilidade de nitrogênio para as minicepas, e o excesso deste nutriente pode ocasionar a redução do enraizamento das miniestacas, além de prejuízo na produtividade das minicepas. O fato de que I. paraguariensis se desenvolve satisfatoriamente em solos de baixa fertilidade é um indicativo de que a espécie não exige altas doses de nutrientes (Carvalho et al. 2003). Entretanto, considerando a alta exportação de material vegetal no minijardim clonal, é recomendável a adequada nutrição das plantas em $\mathrm{N}$, que neste caso apresentou respostas positivas quando foi aplicado em doses de até $380 \mathrm{mg} \mathrm{L}^{-1}$.

\section{Conclusões}

A miniestaquia de erva-mate é influenciada pela época de coleta dos propágulos, disponibilidade de nitrogênio para as minicepas e pelo material genético. Recomenda-se o uso de 104 a 380 mg L-1 de nitrogênio na solução nutritiva das minicepas.

\section{Agradecimentos}

Agradecemos à Coordenação de Aperfeiçoamento de Pessoal de Nível Superior (CAPES) pela concessão de bolsas de estudo.

\section{Referências}

Barbosa JZ, Motta ACV, Consalter R, Poggere GC, Santin D, Wendling I (2018) Plant growth, nutrients and potentially toxic elements in leaves of yerba mate clones in response to phosphorus in acid soils. Anais da Academia Brasileira de Ciencias, 90(1):557-571. doi: 10.1590/00013765201820160701
Brondani GE, Araújo MA, Wendling I, Kratz D (2008) Enraizamento de miniestacas de erva-mate sob diferentes ambientes. Pesquisa Florestal Brasileira, 57:29-38.

Carvalho PER (2003) Espécies arbóreas brasileiras. Colombo: Brasília: Embrapa Informação tecnológica; Colombo: Embrapa Florestas. 1035 p.

Dartora N, De Souza LM, Paiva SMM, Scoparo CT, Iacomini M, Gorin PA, Rattmann YD, Sassaki GL (2013) Rhamnogalacturonan from Ilex paraguariensis: A potential adjuvant in sepsis treatment. Carbohydrate Polymers, 92(2):17761782. doi: 10.1016/j.carbpol.2012.11.013

Duarte MM, Mireski MC, Oliszeski A, Wendling I, Stuepp CA (2019) Rooting of yerba mate cuttings with different lenghts. Revista Eletrônica Científica da UERGS, 5(1):05-11. doi: 10.21674/24480479.51.05-11

Goulart PB, Xavier A (2010) Influência do modo de acondicionamento de miniestacas no enraizamento de clones de Eucalyptus grandis x E. urophylla. Revista Arvore, 34(3):407-415. doi: 10.1590/S010067622010000300004

Hartmann HT, Kerster DE, Davies JR FT, Geneve RL (2017) Plant Propagation: principles and practices. $9^{\text {th }}$ ed. Boston: Prentice Hal. 1024 p.

Kojima M, Kamada-Nobusada T, Komatsu H, Takei K, Kuroha T, Mizutani M, Ashikari M, UeguchiTanaka M, Matsuoka M, Suzuki K, Sakakibara H (2009) Highly sensitive and high-throughput analysis of plant hormones using MS-probe modification and liquid chromatographytandem mass spectrometry: An application for hormone profiling in Oryza sativa. Plant and Cell Physiology, 50(7):1201-1214. doi: 10.1093/pcp/pcp057

Kratz D, Pires PP, Stuepp CA, Wendling I (2015) Produção de mudas de erva-mate por miniestaquia em substratos renováveis. Floresta, 45(3):609-616. doi: 10.5380/rf.v45i3.36531

Nascimento B, Sá ACS, Lemos LB, Rosa DP, Pereira MO, Navroski MC (2018) Three epicormic shoot techniques in I. paraguariensis mother trees ans its cutting according to the material rejuvenation degree. Cerne, 24(3):240-248. doi: $10.1590 / 01047760201824032584$

Rocha JHT, Backes C, Borelli K, Prieto MR, Santos AJM, Godinho TO (2015) Produtividade do minijardim e qualidade de miniestacas de um clone híbrido de Eucalyptus grandis $\mathrm{x}$ Eucalyptus urophylla (I-224) em função de doses de nitrogênio. Ciência Florestal, 25(2):273-279. doi: 


\section{Duarte et al.}

\section{$10.5902 / 1980509818411$}

Sá FP, Portes DC, Wendling W, Zuffellato-Ribas KC (2018) Miniestaquia de erva-mate em quatro épocas do ano. Ciência Florestal, 28(4):1431-1442. doi: 10.5902/1980509835051

Santin D, Benedetti EL, Bastos MC, Kaseker JF, Reissmann CB, Brondani GE, Barros NF (2013) Crescimento e nutrição de erva-mate influenciados pela adubação nitrogenada, fosfatada e potássica. Indian Medicinal Plants, 23(2):363-375. doi: 10.1007/978-0-387-70638-2_777

Santin D, Benedetti EL, Brondani GE, Reissmann CB, Orrutéa AG, Roveda LG (2008) Crescimento de mudas de erva-mate fertilizadas com N, P e K. Scentia Agraria, 9(1):59-66.

Santin D, Wendling I, Benedetti EL, Morandi D, Domingos DM (2015) Sobrevivência, crescimento e produtividade de plantas de erva-mate produzidas por miniestacas juvenis e por sementes. Ciencia Florestal, 25(3):571-579. doi: $10.5902 / 1980509819608$

Somkuwar RG, Bondage DD, Surange MS, Ramteke SD (2011) Rooting behaviour, polyphenol oxidase activity, and biochemical changes in grape rootstocks at different growth stages. Turkish Journal of Agriculture and Forestry, v. 35, n. 3, p. 281-287. doi: 10.3906/tar-0911-62

Stuepp CA, De Bitencourt J, Wendling I, Koehler HS, Zuffellato-Ribas KC (2015) Propagação de erva-mate utilizando brotações de anelamento e decepa em matrizes de duas idades. Cerne, 21(4):519-526.

doi:

$10.1590 / 01047760201521041864$

Stuepp CA, De Bitencourt J, Wendling I, Koehler HS, Zuffellato-Ribas KC (2017) Age of stock plants, seasons and AIB effect on vegetative propagation of Ilex paraguariensis. Revista Arvore, 41(2):01-07. doi: 10.1590/1806-90882017000200004

Stuepp CA, Pereira GP, Zem LM, Peña ML, Bueno PMC, Spader V, Zuffellato-Ribas KC, Rosa GM (2013) Enraizamento de melaleuca: Influência da altura de coleta das estacas e aplicação de IBA. Colloquium Agrariae, 9(1):01-09. doi: 10.5747/ca.2013.v09.n1.a083

Sturion JA, Stuepp CA, Wendling I (2017) Genetic parameters estimates and visual selection for leaves production in Ilex paraguariensis. Bragantia, 76(4):492-500. doi: 10.1590/1678-4499.2016.419

Taiz L, Zeiger E, Møller IM, Murphy A (2017) Fisiologia e Desenvolvimento Vegetal. $6^{\text {th }}$ ed. Porto
Alegre: Artmed. 888 p.

Tronco KMQ, Bisognin DA, Fleig FD, Horbach MA (2015) Enraizamento ex vitro e aclimatização de microestacas de Ilex paraguariensis A. St Hil. Cerne, 21(3):371-378. doi: $10.1590 / 01047760201521031523$

Wendling I, Brondani GE, Dutra LF, Hansel FA (2010) Mini-cuttings technique: A new ex vitro method for conal propagation of sweetgum. New Forests, 39(3):343-353. doi: 10.1007/s11056-0099175-2

Wendling I, Santin D (2015) Propagação e nutrição de erva-mate. Brasília: Embrapa. 195 p.

Xavier A, Wendling I, Silva RL (2013) Silvicultura clonal: princípios e técnicas. $2^{\circ}$ ed. Viçosa: UFV. $272 \mathrm{p}$. 